\title{
Objective Sea-Level Pressure Analysis for Data-Sparse Areas
}

\author{
LEONARD M. DRUYAN \\ Inslitute for Space Studies, Goddard Space Flight Center, NASA, New York, N. Y. 10025
}

(Manuscript received 11 August 1972, in revised form 20 October 1972)

\begin{abstract}
A computer procedure, described in an earlier study, uses the wind speed field near the ocean surface in combination with a small number of observations of pressure and wind velocity to specify the maritime sea-level pressure field. An improved version was used to analyze the pressure distribution over the North Pacific Ocean for eleven synoptic times in February 1967. Independent knowledge of the central pressures of lows is shown to reduce the analysis errors for very sparse data coverage. The application of planned remote sensing of sea-level wind speeds is shown to make a significant contribution to the quality of the analysis especially in the high gradient mid-latitudes and for sparse coverage of conventional observations (such as over Southern Hemisphere oceans). Uniform distribution of the available observations of sea-level pressure and wind velocity yjelds results far superior to those derived from a random distribution. A generalization of the results indicates that the average lower limit for analysis errors is between 2 and $2.5 \mathrm{mb}$ based on the perfect specification of the magnitude of the sea-level pressure gradient from a known verification analysis, and 15 uniformly distributed, high-quality buoy, weather ship or island observations of the pressure and wind velocity. (A less than perfect specification will derive from wind-pressure relationships applied to satellite-observed wind speeds.) Analysis errors computed using poorly defined wind fields indicate the procedure's potential for sparse data analysis even without supplementary satellite data.
\end{abstract}

\section{Introduction}

A previous paper by the author (Druyan, 1972) has described a computer procedure for the analysis of maritime sea-level pressure and wind fields. The method is an application of the satellite system proposed by Moore and Pierson (1970) for the remote measurement of sea-level wind speed.

This paper is based on an improved version of the procedure; it discusses the analysis sensitivity to the quality and distribution of conventional observations and the quality of the pressure gradient specification.

Research is continuing on the experimental and theoretical aspects of using active microwave radar and passive radiometry to measure the energy in that portion of the ocean wave spectra which is the most sensitive to the local wind (Pierson and Moore, 1972; Pierson et al., 1971). A prototype instrument will be operated from the manned orbiting satellite, Skylab, and may collect data of this type from space for the first time in the spring of 1973.

Better specification of the sea-level pressure and wind fields has obvious application to ocean wave forecasting and weather forecasting for coastal regions. In addition, the combination of satellite-derived temperature profiles with sea-level pressures can serve to improve the specification of upper pressure surfaces through the hydrostatic formulation. Several numerical simulation studies also indicate that wind errors may be more effectively reduced by inserting temperature profile data in combination with sea-level pressures rather than with pressure data from a near-tropopause level (Kasahara and Williamson, 1972; Jastrow and Halem, 1973). Moreover, because of the vast ocean areas of the Southern Hemisphere, supplying surface pressures only over land gives considerably larger circulation errors than when the entire reference pressure field is specified (Jastrow and Halem, 1973).

\section{General discussion of experiments}

A large number of observations of sea-level wind and pressure (hereafter referred to as the conventional observations) over the North Pacific Ocean was gathered from non-real-time sources to supplement data normally available at map time. [A discussion of the availability and reliability of these data is found in Cardone (1969).] A horizontal interpolation of the wind field according to Cardone's model of the marine boundary layer yields a gridded array of wind speeds consistent with the 200 or more conventional observations of a given map time (see Appendix). These speeds are used to simulate the pattern of wind speed data that would become routinely available should the proposal of Moore and Pierson prove feasible. [A more detailed discussion of the simulation of satellite-observed data and the recovery of the entire wind speed field is found in Druyan (1972).]

The input for each analysis is a small number of conventional meteorological observations, the location 


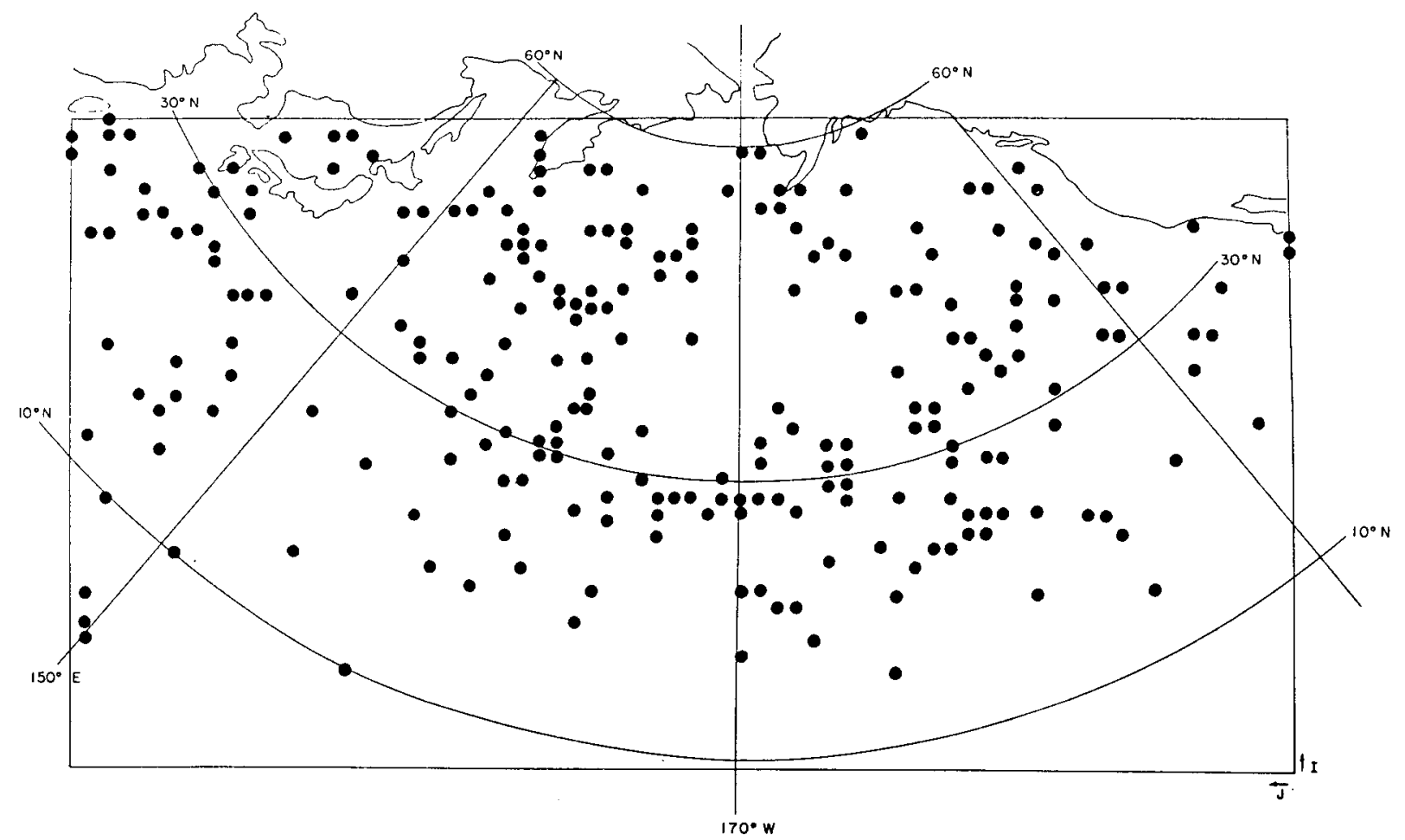

FIG. 1. Extent of area under study. The borders of the Cartesian grid system are represented and the dots indicate the locations of the maritime observations collected for 8 February 1967.

of low pressure centers, and the extensive specification of the sea-level wind speed field. The procedure uses the wind speed in order to estimate the pressure gradient magnitude, and the pressure field is extrapolated outward from the low pressure centers and the conventional observations.

Persistence or even climatology often provides a realistic analysis of the sea-level pressure field in the tropics. On the other hand, extrapolating the midlatitude pressure analysis to very low latitudes using wind-pressure relationships is a precarious venture. Unfortunately, the grid-by-grid specification of such tropical pressures is tedious to prepare for the particular grid system used in this study. Therefore, observations from eight tropical stations, some corresponding to islands, one to weather ship $\mathrm{N}$, and the others estimated from the real-time NMC Northern Hemisphere analysis, were used as permanent input data. Also, in each case an observation was added in the area of Los Angeles since a considerable number of grid points lie over the western United States.

In order to integrate the analysis with the coastal pressure observed by continental stations, pressures are made available to the analysis along the northernmost $i$ grid line (see Fig. 1), and three horizontal rows of pressure are interpolated between these border values and those from within the analysis.

The boundary data and the tropical observations are referred to as the "permanent data" in the discussion.
The central pressure of each low acts as a seed in determining the value of the pressures within its area of influence. Each such pressure minimum is computed as the distance-weighted average of estimates derived from conventional pressure and wind velocity observations and/or northern boundary pressure values located within some scan radius from the low pressure center. The analysis procedure modifies the initially symmetric pattern of pressures increasing radially outward from each low center by causing the isobars to separate or merge in conformity with the wind-speed-implied magnitude of the pressure gradient.

Similarly, the analysis in the vicinity of an observing station uses the observed pressure as the seed value; the total pressure gradient at the point of observation is determined by the wind velocity. The procedure extends the analysis outward from each such observation by allowing the isobar spacing to adjust to the wind-derived pressure gradient magnitude at each surrounding grid point.

The procedure has been slightly improved since the initial results were published (Druyan, 1972). A significant amount of computer program debugging has eliminated the infrequent appearance of spurious pressures at several grid points in the analysis. Further, whereas it was earlier reported that the subtropical high pressure ridges were located by searching for the highest pressure observation in each low pressure system quadrant, the newer version predesignates the 
latitude of this ridge by climatological considerations. Other high pressure centers result either from a corresponding pressure observation or from the relative maximum created between two adjacent low pressure systems.

A pressure field derived from all of the available conventional observations at a given map time is used as the standard for the verification of a data-sparse analysis; grid-by-grid pressures are compared and the rms difference between the two fields is referred to as the analysis error.

For the purposes of creating a smooth pressure field for verification, consistent with a high density of observations (see, for example, Fig. 1), it was found that an analysis method different from the procedure under discussion (which shows promise for situations of very sparse observational coverage) was desirable. The pressures for each verification field are assigned by taking a distance-weighted average of estimates computed for each grid point from the pressure and wind observations within some scan radius from the grid point. In addition to improving the all-data map, the new procedure also makes the verification more meaningful because the fields to be compared are created by independent procedures.

Sea-level pressure analyses for the North Pacific Ocean (see Fig. 1) were made for eleven synoptic times in February 1967, using, in each case, the permanent data and either all of the available conventional observations or a data set representing a sparse network of observations. The analysis error was computed each time and averaged over the eleven maps for each experiment.

\section{Sensitivity to central pressure}

Since the analysis technique uses low pressure centers as starting points for much of the extrapolation, the objective estimate of the central pressures is crucial to the accuracy of the resulting field. Under operational circumstances, the central pressure can often be estimated from extrapolation in time to within a few millibars accuracy; otherwise, on-time observations from nearby land or maritime stations are needed to make these estimates.

In order to determine the extent to which a "perfect" knowledge of the central pressures can improve the accuracy of the analysis, the eleven pressure fields were generated with various distributions of the conventional meteorological network with the values of the pressure minima being specified externally. (Those computed for the corresponding maximum data analyses were used.) Fig. 2 shows the two graphs of the average analysis errors for the eleven synoptic times plotted as a function of the number of uniformly distributed conventional observations, one for the analyses which were supplied with the central pressures and the other for the analyses which computed these values

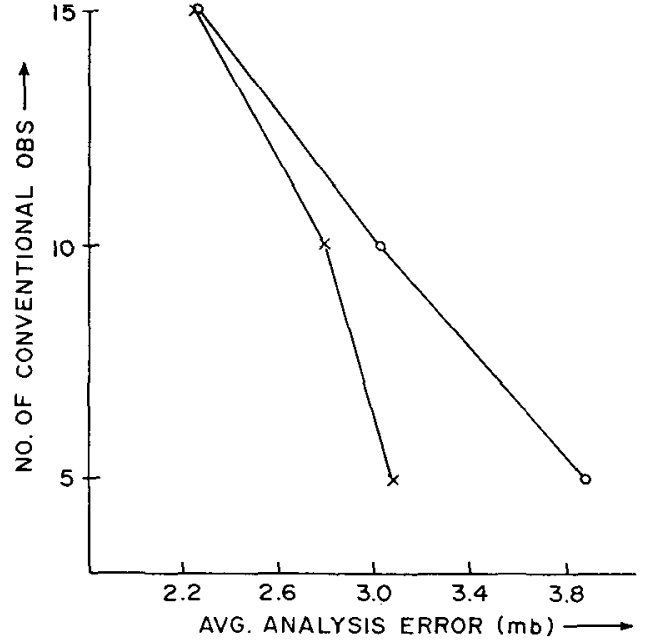

Fig. 2. Average analysis errors for eleven synoptic times in lebruary 1967 plotted as a function of the number of uniformly distributed observations. The analyses represented by both curves used well-specified wind speed fields in order to compute the pressure gradient, but one set $(X)$ was supplied with perfect central pressures while the other $(O)$ computed them internally.

internally. Although the average analysis error can be reduced by as much as $1 \mathrm{mb}$ by supplying the values of the pressure minima to analyses based on five meteorological stations over the area, the advantage almost disappears when as many as 15 well-deployed stations are available. In the latter circumstance, the objective determination is quite adequate.

\section{Sensitivity to the pressure gradient formulation}

Given a "perfect" estimate of the central pressure and the magnitude of the pressure gradient, any remaining deficiencies of the resulting analysis are due to inadequacies in the objective parameterization of the gradient direction. A "perfect" field was specified for each of the eleven synoptic times by using the analysis of the hundreds of conventional meteorological observations as discussed above; "perfect" central pressures and pressure gradients were thus created from these fields.

Using these central pressures and gradients as well as the permanent data, but with none of the original conventional observational data, each of the eleven fields was recovered. The results were compared to the original all-data fields by computing the rms difference between their pressure at corresponding grid points. The average analysis error for the eleven maps is 3.2 mb. This "residual" error can be directly attributed to errors in the gradient direction, not explicitly assigned at each grid point; it is, of course, independent of errors in the magnitude of the gradients and errors in the "seed" or "anchor" pressures. On the other hand, the inclusion of the conventional observations, as in many of the analyses discussed below, somewhat limits the extent of errors caused by estimates of the gradient direction. 

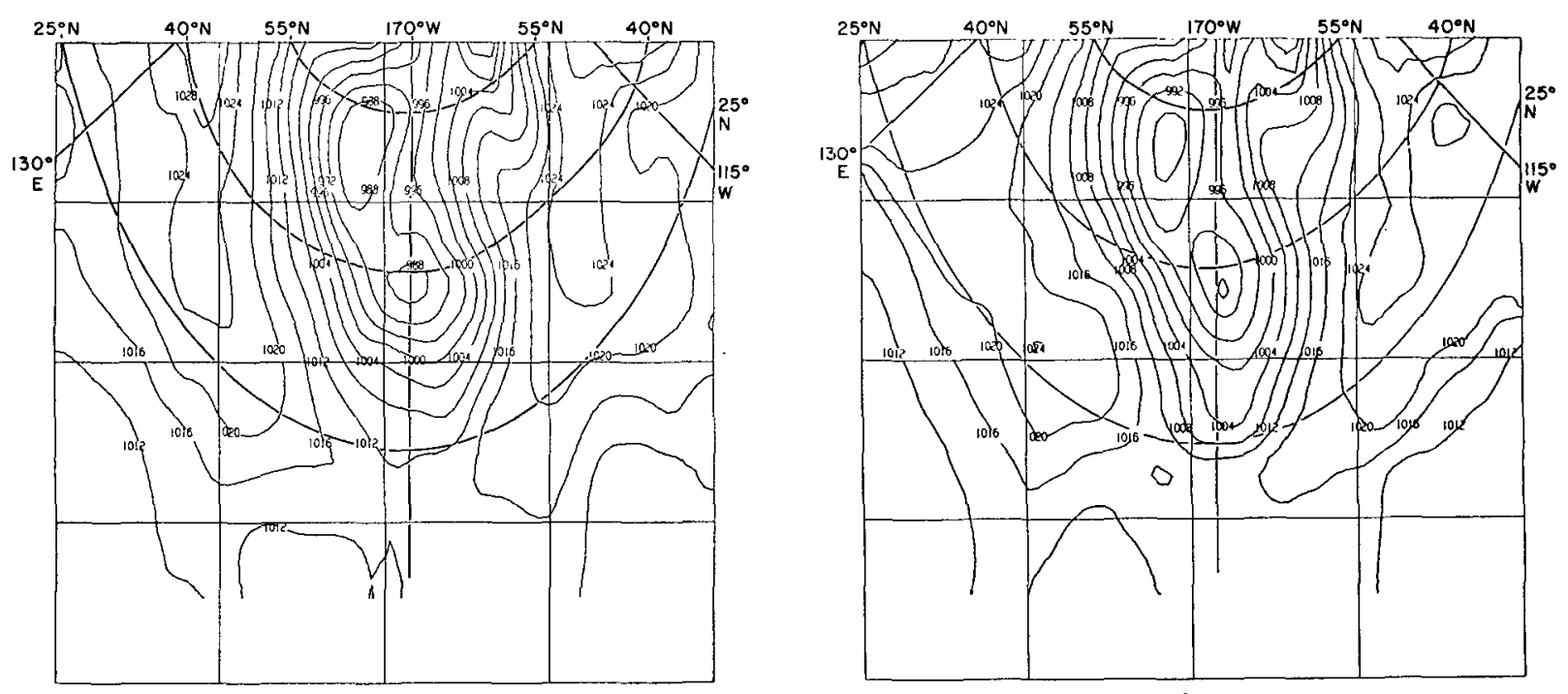

a.
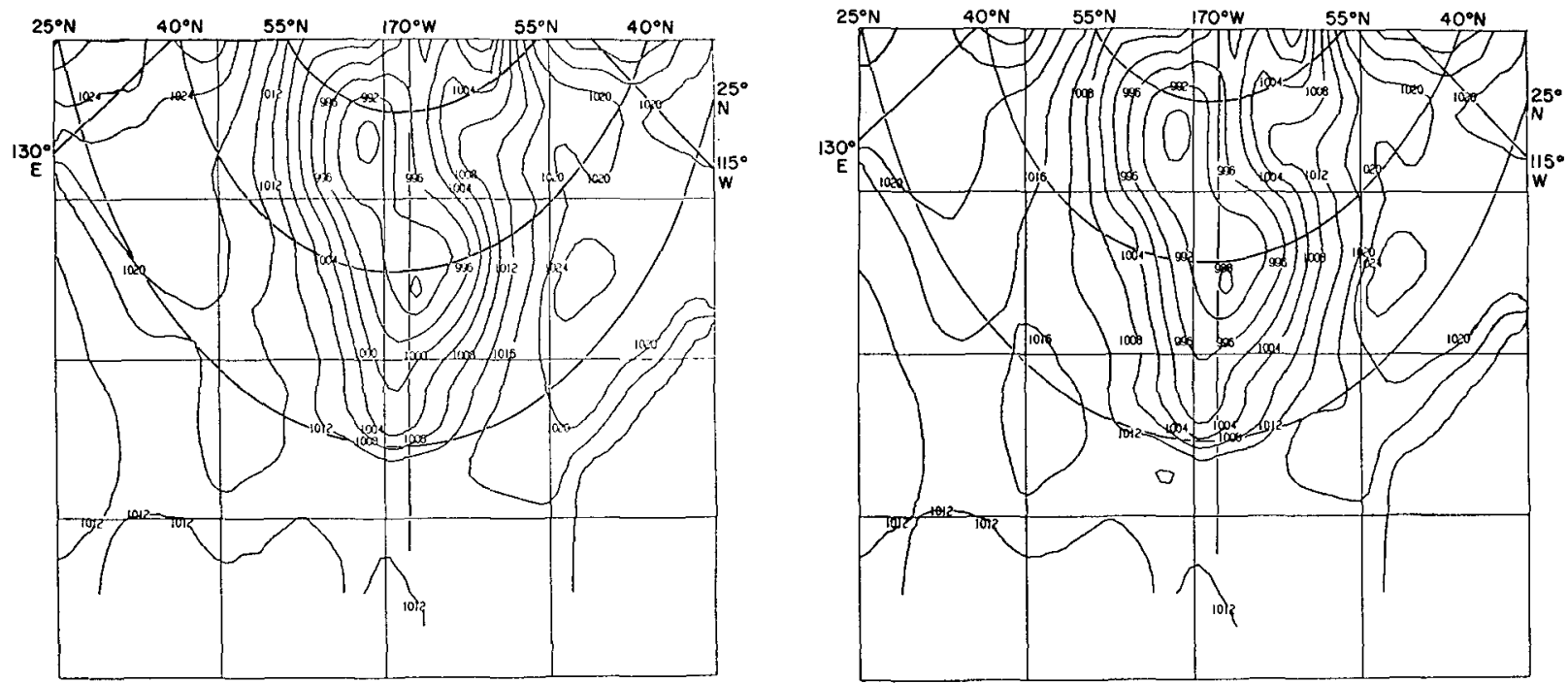

c.

d.
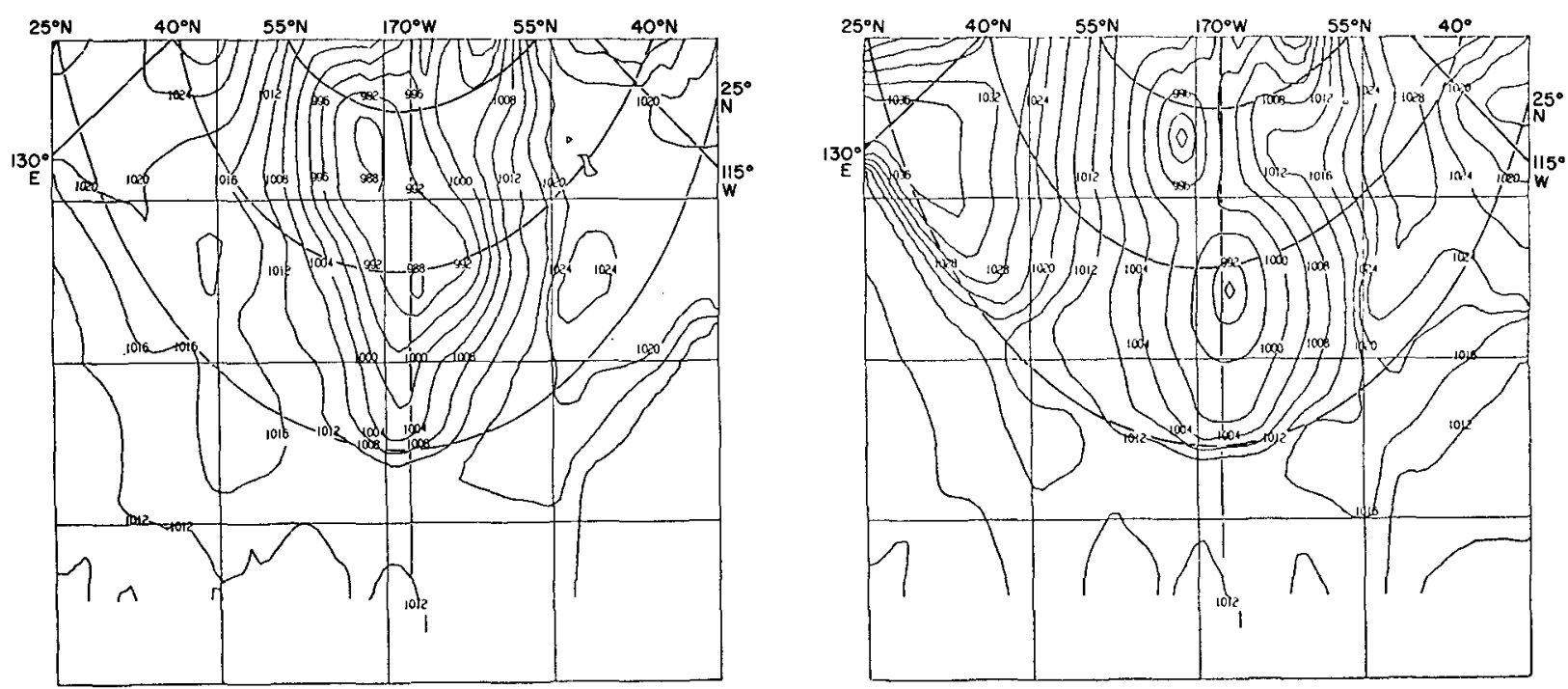

e.

f. 
The pressure gradient magnitudes were then computed by objectively analyzed wind speeds according to the formulation discussed in detail in the original study cited above (see also Appendix). The average analysis error for the same eleven fields analyzed as before, save for the use of the wind speeds in the computation of the gradients, was slightly more than $\frac{1}{2} \mathrm{mb}$ larger than that computed above for the perfect gradient analyses. Thus, if these maps are representative of winter situations, it may be concluded that the analysis is degraded by about $\frac{1}{2} \mathrm{mb}$ in the rms sense, due to the deviation of the pressure gradient magnitude from even a well-specified wind speed field as compared to the ideal specification of "perfect" gradients. Limitations in the objective wind analysis of Cardone (1969) and in the particular calculation of the pressure gradient magnitude [the equations, which were reported in the original study (Druyan, 1972), represent a series of step functions and thus depart from Cardone's intended formulation of the dependence of the observed wind on pressure gradient, air-sea temperature difference, and latitude (see Appendix)] can eventually be overcome and may thus contribute to a reduction of this particular source of error.

The scheme whereby the wind speed is used to compute the magnitude of the pressure gradient involves an iteration of the pressure analysis. The pressure field after the first iteration is used to compute isobar curvature which modifies the previously determined pressure gradients before the second iteration of the analysis is executed. In order to determine whether or not the time-consuming iteration makes a significantly positive contribution to the accuracy of the analysis, the eleven fields were verified before the second iteration in which the pressure gradients are modified by curvature. The average analysis error computes to more than $\frac{1}{2} \mathrm{mb}$ higher than when the effect of curvature is included.

The experiment was repeated with vet another formulation of pressure gradient magnitude. A set of random wind speed errors with a standard deviation of $4.6 \mathrm{kt}$ was generated. These were used to contaminate the wind speed field in random fashion by adding or subtracting them from the objectively analyzed values used in the previous experiment. The resulting "degraded" wind speeds were then used as before to compute the pressure gradient magnitudes. The average analysis error of the resulting eleven sea-level pressure fields was $4.0 \mathrm{mb}$, about $0.8 \mathrm{mb}$ greater than that incurred by using perfect pressure gradients and only about $0.25 \mathrm{mb}$ greater than that incurred by using the original wind speed analysis. Undoubtedly the smoothing of the final field eliminates much of the error produced by the random contaminations. It is also probable

that the original wind speed field contained its own random errors.

In order to determine the analysis errors that would result from a "worst guess" wind speed specification, the eleven sea-level pressure fields were analyzed by assuming a wind speed at every grid point of $15 \mathrm{kt}$ and computing the pressure gradient as before. Thus, the pressure gradient magnitudes varied only according to latitude (due to the latitudinal dependence of the Coriolis parameter) and air-sea temperature difference (see I)ruyan, 1972). The average analysis error of the resulting fields was $5.5 \mathrm{mb}$ which is almost $2 \mathrm{mb}(48 \%)$ higher than that resulting from the well-specified wind speeds and about $2.3 \mathrm{mb}$ greater than that incurred by the perfect pressure gradient formulation. Accounting for curvature provided no significant systematic improvement in the analyses based on 15-kt wind speed.

It should be noted that the average analysis errors given above include the well-specified (by the permanent data), low-gradient tropics as well as the highgradient, mid-latitude regions. The errors are always lower in the former and larger in the latter area than the rms values computed for the entire field. Also, the deterioration due to a less realistic pressure gradient formulation is more pronounced in the mid-latitudes than in the tropics. The average rms error computed for only the northern half of each map is given for the different formulations of the pressure gradient:

Perfect pressure gradient

Well-specified wind speeds

Well-specified wind speed, no curvature

Perturbed wind speeds

Uniform 15-kt wind speeds

Fig. 3 shows five sample sea-level pressure analyses based on each of the above formulations of the pressure gradient. (There is a considerable distortion in these computer-drawn maps which have stretched the vertical dimension relative to the horizontal. For a true representation of the field dimensions see Fig. 1.) Also shown is the verification map (Fig. 3a) which represents the analysis of about 250 ship observations for 0000 GMT 8 February 1967. The analysis which assumed the perfect pressure gradients (Fig. 3b) has reproduced the actual field to a remarkable extent considering how few pressure values were supplied externally (the permanent data and the central pressures of lows). On the other hand, the analysis which assumed a 15-kt wind speed at each grid point (Fig. 3f) has distorted the pattern on the western side of the low pressure trough, has completely missed the narrow ridge in the west and has grossly overestimated the pressures at the upper left edge.

Fig. 3. The sea-level pressure fields for 8 February 1967 isoplethed automatically and objectively analyzed: a. for about 250 actual ship observations of pressure and wind. All remaining fields include perfect central pressures with the following additional features : $b$. perfect pressure gradients; analysis error $=2.2 \mathrm{mb}$. $c$. a well-specified wind speed field; analysis error $=2.7 \mathrm{mb}$. d. same as c. but neglecting curvature; analysis error $=3.5 \mathrm{mb}$. e. a wind speed field degraded by random errors; analysis error $=3.0 \mathrm{mb}$. $\mathrm{f}$. a uniform wind speed of $15 \mathrm{kt}$; analysis error $=5.1 \mathrm{mb}$. (Note that the vertical dimension has been stretched in these computergenerated representations.) 


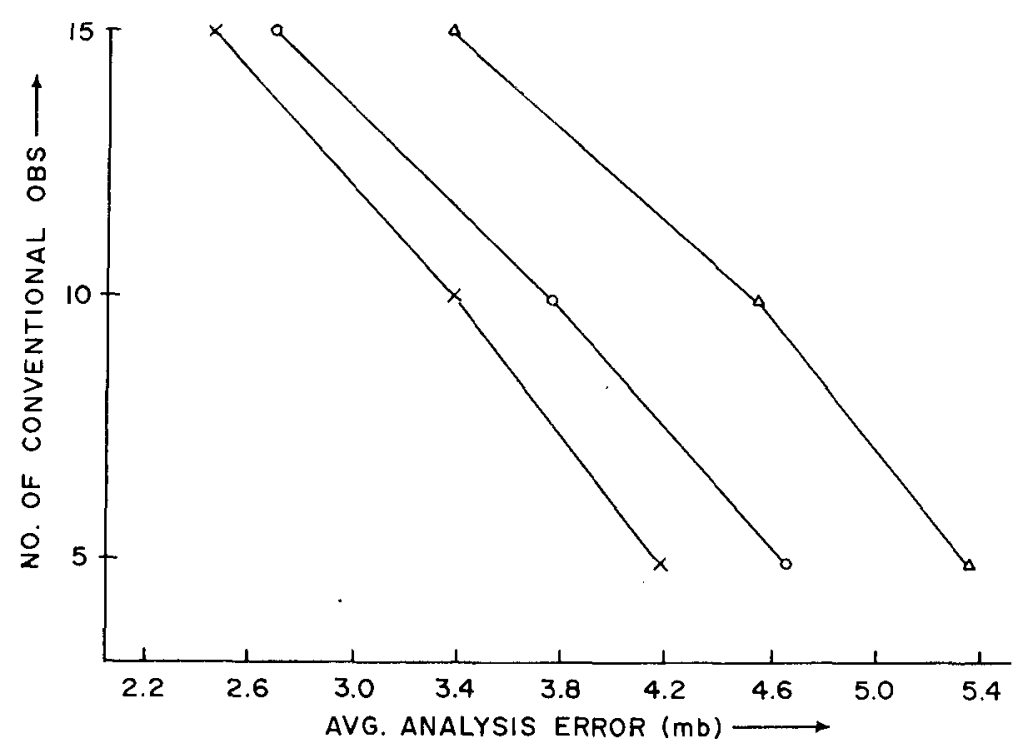

FIG. 4, Average analysis errors for the northern half plotted as a function of the number of uniformly distributed observations. The eleven sea-level pressure fields are the same as those whose analysis errors are shown in Fig. 2, but the central pressures have in every case been computed internally and the pressure gradient has been either specified from the corresponding perfect analyses $(X)$, computed from well-specified wind speeds $(O)$, or computed by assuming a 15-kt wind speed everywhere $(\Delta)$.

Of the three remaining charts in Fig. 3, the one which used the well-specified wind speed field and corrected for curvature $(3 \mathrm{c})$ came the closest to building the narrow ridge in the west to proper strength. The analysis for which curvature was neglected (3d) is slightly inferior to that of $3 c$. The analysis which used the contaminated wind speeds ( $3 \mathrm{e}$ ) erroneously widened the trough between the two low centers and completely missed the short-wave ridge outlined by the 1004-mb isobar on all of the other maps northeast of the elongated low pressure trough. All three of these maps missed the ridging in the east.

Fig. 4 shows three graphs of the average analysis errors of the eleven synoptic times computed for the northern half of the analysis area and plotted as a function of the number of uniformly distributed conventional observations for different formulations of the pressure gradient. The perfect pressure gradient derived analyses verify with lower average errors than those based on the wind speed field at all observational network sizes. The deterioration of the analysis quality is, of course, much more pronounced when uniform wind speeds of $15 \mathrm{kt}$ are assumed.

\section{Sensitivity to the quality and distribution of conventional observations}

The sensitivity of the analysis to the quality and distribution of conventional meteorological observations was tested. The maximum data analyses were used to simulate "perfect" observations of sea-level pressure and wind velocity at designated grid points.
These grid points represent a plausible buoy or ship network at which the meteorological observations are assumed to be of high quality.

Any given number of observations was deployed in one of three alternative distributions: a network representing uniform. (within the limitations of the number) buoy coverage of the non-tropical areas, a northern shift which concentrated the stations in the northern third of the field but nevertheless maintained uniform spacing between them, and a random distribution where uniform spacing was not guaranteed. The eleven sets of analyses were completed by using each of the observational networks in combination with the wellspecified wind speed fields; the pressure minima were computed objectively from the available data. The average analysis errors incurred by each network for the eleven maps are plotted as a function of the number of observations in Fig. 5.

The use of the uniform distribution of buoys resulted in consistently superior analyses although the central pressure was determined objectively by the model. The advantage afforded the central pressure computation by the high density of observations in the region of maximum cyclone activity provided by the northernmost distribution as well as the strategic value of having more observations over the high gradient region were apparently offset by data voids elsewhere.

Coordinates were chosen by random selection in order to represent a network of ships whose meteorological observations are of superior quality. These latter were simulated in the same manner as the "buoy" 
obscrvations above. In each of three independent random selections of 15 observations, the first 5 , the first 10, and all 15 were rach respectively designated ats observational networks.

The average analysis error for the eleven maps for a given number of "ships" was averaged for the three different randomly picked distributions. The analysis errors were consistently higher than their counterparts computed from a uniformly distributed network of buoy observations (see Fig. 5 ). The considerable variability observed in the quality of these analyses indicates the extreme sensitivity of the procedure to the deployment of conventional observations.

Three independent random picks were made of 15 actual ship reports, each from the entire list of observations available for each of the eleven maps. As before, their deployment over the analysis area is also completely random but, in this case, it varies from map to map. All 15 observations, the first 10 and the first 5 for each of the three random selections determined an observational network for analysis. Whereas the simulated observations were derived from each smooth alldata "perfect" analysis, the individual ship observations of sea-level pressure and wind are sometimes less representative of the "perfect" analysis against which the results are verified. This "perfect" pressure field represents the integration of data from a high-density network and, as such, the impact of any one observation that is "out of step" with the majority in its vicinity is diminished. However, when such an observation is used in a sparse data analysis, its sphere of influence is quite extensive.

As before, the average analysis error for the eleven maps for each of the three network sizes was averaged for the three different random selections. The grand average rms obtained for the 15-ship network is 4.0 $\mathrm{mb}$; for the 10-ship network it is $4.2 \mathrm{mb}$; and for the 5 -ship network it is $6.0 \mathrm{mb}$ (see Fig. 5).

The results reflect sensitivity of the analyses to the randomness of the distribution and to the non-representativeness of some of the observations. Because of the latter, these analyses are inferior to those obtained for the random distribution of "perfect" observations and they are more inferior yet when compared to the "perfect" and uniform-distribution derived maps.

\section{Conclusions}

Sea-level pressure fields generated by the procedure are shown to be of higher quality when the pressure at the low centers is supplied externally in combination with five uniformly distributed, perfect observations of pressure and wind than when these pressure minima are computed internally. However, this advantage disappears when as many as 15 observations are used.

The computation of the pressure gradient from a well-specified wind speed field, which could become a routine product of satellite observation if current research bears fruit, yields analyses which are, on the average, superior to those which are deprived of the wind speed specification. This deterioration is reflected by an increase in the average analysis error for the northern half of $2.5 \mathrm{mb}$ in those charts which were generated from the "seed" pressures at the low centers. The amount of the error increase due to poor wind speed specification is somewhat diminished by pressure observations which correct the analysis wherever they are made available. Smoothing of the pressure analysis

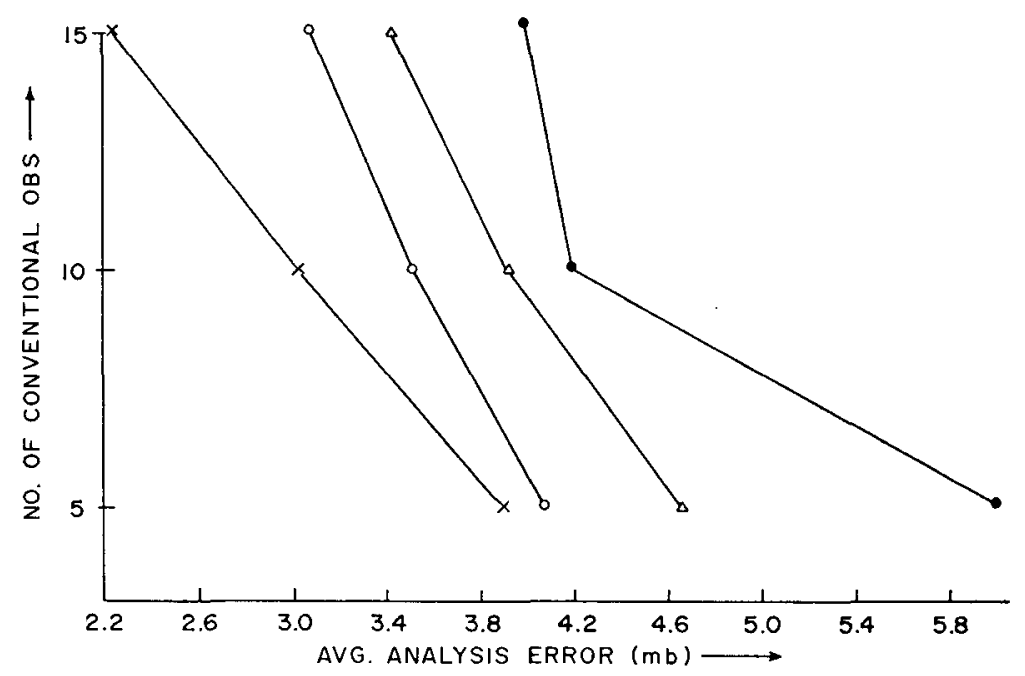

Fig. 5. Average analysis errors for the eleven maps plotted as a function of the number of observations. All analyses computed the pressure minima internally and the pressure gradients from well-specified wind speed fields. The observations simulate a uniform network of buoy stations $(X)$, a northern shift network of buoy stations $(O)$, and a random distribution of high quality ship observations $(\Delta)$; the remaining curve $(\bullet)$ shows the average analysis errors resulting from the random distribution of actual ship observations. 
greally reduces the inpact of random errors in the wind speed field and thus the charts generated from such fields are only slightly inferior to those based on a good wind speed specification. Accounting for curvature by iterating the analysis a second time appears to have a significantly positive effect except when uniform speeds are used. The improvement in the analyses resulting from specifying perfect pressure gradient magnitudes indicates that better results can be expected from more representative wind speed fields and better transformation from wind speeds to pressure gradients.

Uniform spacing of the observation network is shown to be far superior to the use of random maritime observations even when the latter are of high reliability; the case for meteorological buoy stations is obvious.

The rather small rms errors computed for the northern half of the field (see Fig. 4) with just 10 or 15 observations suggests the model's possible application to sea-level pressure analysis in data-sparse regions even without the availability of satellite-observed wind speeds. The analyses based on $15 \mathrm{kt}$ yield an upper limit to the error since some climatological guess of the wind field should be superior to the uniform speed assumption. Traditional analysis procedures depend on a sometimes poor initial guess field which is barely modified in data-sparse regions; the one described here constructs a completely new field by the interpolation between, and extrapolation from, known observations. Lewis and Grayson (1972) have demonstrated how a low error initial guess field can be improved by considering supplemental wind data, but the construction of a new pressure field, consistent with the available wind data as described above, provides a promising alternative to the traditional correction methods.

The isobar orientation implies a wind direction and, in combination with an observed wind speed field, defines the total wind vector. Investigation into the specification of the sea-level wind velocity derived from the data-sparse analyses is proceeding and application of the results to ocean wave forecasting will be considered.

Acknowledgments. The research described in this study was performed during the author's tenure as Research Associate with the support of the National Academy of Science-National Research Council. The facilities provided by Dr. Robert Jastrow at the Goddard Institute for Space Studies are gratefully acknowledged. The author is indebted to Prof. W. J. Pierson of New York University for his continuous interest and encouragement of the research. The author would also like to thank Prof. V. J. Cardone of New York University for his help in the preparation of the Appendix.

\section{APPENIIX}

\section{Summary of the Cardone (1969) Marine Boundary Layer Model}

\section{The relationship between the geostropic and actual sea-level winds}

The Cardone formulation is an extension of Blackadar's (1965) two-layer modeling of the boundary layer wind profile. It assumes a constant-flux layer in which the eddy viscosity variation with height is specified as a function of the atmospheric stability according to the Monin-Obukov similarity theory and an upper layer which incorporates an Ekman profile with a constant eddy viscosity. The characteristics of the lower boundary are prescribed internally by a functional relationship between the roughness parameter $Z_{0}(\mathrm{~cm})$ and the friction velocity $U_{*}\left(\mathrm{~cm} \mathrm{sec}^{-1}\right)$ :

$$
Z_{0}=\left(0.684 / U_{*}\right)+4.28 \times 10^{-5} U_{*^{2}}-4.43 \times 10^{-2} .
$$

The following set of simultaneous equations relating the geostrophic wind $(G)$ and the air-sea temperature difference $\left(\theta_{a}-\theta_{s}\right)$ with the stability length $\left(L^{\prime}\right)$, the cross-isobar angle of the wind vector $\left(\Psi_{0}\right)$, and $U_{*}$ are solved by numerical iteration, i.e.,

$$
\left.\begin{array}{rl}
L^{\prime} & =U_{*}^{2} \bar{\theta}\left[\ln \left(Z_{a} / Z_{0}\right)^{\prime}-\Psi\left(Z_{a} / L^{\prime}\right)\right] /\left[K^{2} g\left(\theta_{a}-\theta_{s}\right)\right] \\
U_{*} & =G\left[2 K B_{0} \sin ^{2} \Psi_{0} \phi_{u}\left(h / L^{\prime}\right)\right]^{\frac{1}{2}} \\
U_{*} & =G K \sqrt{2} \sin \left(\pi / 4-\Psi_{0}\right) /\left[\ln B_{0} R_{0}-\Psi\left(h / L^{\prime}\right)\right]
\end{array}\right\},
$$

where:

$h \quad$ height of the top of the lower layer $\left[=B_{0} G / F\right]$

$B_{0} \quad$ dimensionless constant $\left[=3.0 \times 10^{-4}\right]$

$F \quad$ Coriolis parameter $[=2 \Omega \sin (\mathrm{LAT})]$

Ro surface Rossby number $\left[=G / F Z_{0}\right]$

$K \quad$ von Kármán's constant $(\sim 0.4)$

$Z_{a} \quad$ height above the sea surface at which $\theta_{a}$ is taken

$\bar{\theta} \quad$ mean potential temperature of the boundary layer

$\phi_{u}\left(h / L^{\prime}\right)$ non-dimensional wind shear given by $1+\beta^{\prime}$ $\left(h / L^{\prime}\right)$ for stable conditions (i.e., $\left.\theta_{a}>\theta_{s}\right) ; \mathrm{by}$ the implicit so-called KEYPS function (Panofsky, 1963) for unstable conditions, i.e., $\phi_{u}{ }^{4}-\gamma^{\prime} h /\left(L^{\prime} \phi_{u}{ }^{3}-1=0\right) ;$ and $\phi_{u}=1$ for neutral conditions. The constants $\beta^{\prime}$ and $\gamma^{\prime}$ are estimated to be 7 and 18 , respectively.

$\Psi\left(Z / L^{\prime}\right)$ integrated non-dimensional wind shear

$$
\left\{=\int_{0}^{Z / L^{\prime}}\left[1-\phi_{u}(\xi)\right] / \xi d \xi\right\} .
$$

The wind speed at $19.5 \mathrm{~m}$ above the sea surface is $U_{19.5}=\left(U_{*} / K\right) \ln 19.5 / Z_{0}$; the direction of the wind is found at $\Psi_{0}$ degrees from the geostrophic direction.

Thus, the wind vector at $19.5 \mathrm{~m}$ is determined by a numerical iteration from some first guess values of $U_{*}$ and $\Psi_{0}$ as well as the computed geostrophic wind and air-sea temperature differences. 
The problem of determining the pressure gradient from satellite observations of the sea-level wind speed ${ }^{1}$ is the inverse of the Cardone procedure. Thus, the linear relationships presented in Table 1 of Druyan (1972) represent best fits to a wide range of values of $U_{19.5}$ vs $G$ generated over a range of air-sea temperature differences and latitudes by Cardone's scheme.

\section{The objective interpolation of the sea-level winds}

The procedure described above assigns a wind at each point in a field based on the analysis of sea-level pressure and air-sea temperature difference. It is desirable to correct the analysis with observations of the wind that might be available not only at the grid points corresponding to them but also at surrounding points to insure a realistic and smooth field.

Cardone uses the Conditional Relaxation Analysis Method developed by Harris et al. (1966) to integrate the wind observations with those derived from the pressure and temperature field. The latter serve as both boundary values along the edge of the grid array to be computed and as the initial guess field within the array which determines the forcing function for the relaxation. The relaxation is made for the two orthogonal wind components separately and the grid values are required to satisfy Poisson's equation

$$
\left.\begin{array}{l}
\nabla^{2} u(i, j)=F_{u}(i, j) \\
\nabla^{2} v(i, j)=F_{v}(i, j)
\end{array}\right\},
$$

subject to the constraints of the observations of $u$ and $v$ acting as internal boundary points; $F_{u}(i, j)$ and $F_{v}(i, j)$, the forcing functions, define the shape of the

${ }^{1}$ 'The author takes $U_{10.5}$ to represent the sea-level wind speed. $u$ and $v$ fields, respectively, and are computed to be the Laplacian at each $(i, j)$ of the initial guess $u$ and $v$ fields.

For the purposes of this study, such objective wind analyses were converted to gridded arrays of the wind speed which were used to simulate satellite-observed winds within the sub-satellite swaths.

\section{REFERENCES}

Blackadar, A. K., 1965: A simplified two-Jayered model of the baroclinic neutral atmospheric boundary layer. Final Report: Flux of Heat and Momentum in the Planetary Boundary Layer of the Atmosphere, Pennsylvania State University.

Cardone, V., 1969: Specification of the wind distribution in the marine boundary layer for wave forecasting. GSL Rept. TR-69-1, Dept. Meteor. Oceanogr,, New York University.

Druyan, L., 1972: Objective analysis of sea-level winds and pressures derived from simulated observations of a satellite radar-radiometer and actual conventional data. $J$. $A p p l$. Meteor., 11, 413-428.

Harris, R. G., A. Thomasell and J. Welsh, 1966: Studies of techniques for the analysis and prediction of temperature in the ocean. Part III, Automated analysis and prediction. Rept. 7421-213, Travelers Research Center, Inc.

Jastrow, R., and M. Halem, 1973: Simulation studies and the design of the first GARP global experiment. Bull. Amer. Meteor. Soc., 54, 13-21.

Kasahara, A., and D. Williamson, 1972: Evaluation of tropical wind and reference pressure measurements-numerical experiments for observing systems. Telluts, 24, 100-115.

Lewis, J., and T. Grayson, 1972: The adjustment of surface wind and pressure by Sasaki's variational matching technique. J. A ppl. Meleor., 11, 585-597.

Moore, R., and W. Pierson, 1970: Worldwide oceanic wind and wave predictions using a satellite radar-radiometer. AIAA Paper No. 70-310.

Panofsky, H. A., 1963: Determinations of stress from wind and temperature measurements. Quart. J. Roy. Meteor. Soc., $89,85-94$.

Pierson, W., and R. Moore, 1972: The extrapolation of laboratory and aircraft radar sea return data to spacecraft altitudes. Proc. Fourth Annual Eartl Resources Review, Manned Spacecraft Center, Houston, Tex. (in press).

--, I. Jackson, R. Stacy, and E. Mehr, 1971: Research on the problem of the radar return from a wind roughened sea. Advanced Applications of Flight Experiments-Principle Investigators' Review, NASA Langley Research Center, 83-114. 